\title{
\begin{tabular}{l|l} 
Mitraries & DSpace@MIT
\end{tabular}
}

\author{
MIT Open Access Articles
}

Spectral-efficiency of multi-antenna links in
ad-hoc wireless networks with limited Tx CSI

The MIT Faculty has made this article openly available. Please share how this access benefits you. Your story matters.

Citation: Govindasamy, Siddhartan, Daniel W. Bliss, and David H. Staelin. "Spectral-efficiency of Multi-antenna Links in Ad-hoc Wireless Networks with Limited Tx CSI." Conference Record of the Forty-Third Asilomar Conference on Signals, Systems and Computers 2009. 1714-1718. (C) Copyright 2009 IEEE

As Published: http://dx.doi.org/10.1109/ACSSC.2009.5469713

Publisher: Institute of Electrical and Electronics Engineers (IEEE)

Persistent URL: http://hdl.handle.net/1721.1/73108

Version: Final published version: final published article, as it appeared in a journal, conference proceedings, or other formally published context

Terms of Use: Article is made available in accordance with the publisher's policy and may be subject to US copyright law. Please refer to the publisher's site for terms of use. 


\section{Spectral-Efficiency of Multi-antenna Links in Ad-hoc Wireless Networks with Limited Tx CSI}

\author{
Siddhartan Govindasamy \\ Franklin W. Olin College of Engineering \\ siddhartan.govindasamy@olin.edu
}

\author{
Daniel W. Bliss ${ }^{\dagger}$ \\ Massachusetts Institute of Technology
}

\author{
David H. Staelin \\ Research Laboratory of Electronics \\ Massachusetts Institute of Technology \\ staelin@mit.edu
}

\begin{abstract}
An asymptotic expression is derived for the mean spectral efficiency of multi-antenna links randomly distributed on a plane in a uniform manner where transmitters (Tx) have Channel-State-Information (CSI) of the channels between themselves and their target receivers while receivers (Rx) have CSI between themselves and all transmitters. The mean per-link spectral efficiency in the network is found to increase if nodes transmit using a subset of their strongest channel modes instead of the link-optimal strategy of water-filling on all channel modes (Farrokhi et al.). For reasonable parameters, this system which requires limited Tx CSI, has approximately double the mean spectral efficiency of systems without Tx CSI.
\end{abstract}

Index Terms-MIMO, Wireless Networks, Antenna Arrays, Stochastic Geometry, Ad-hoc networks.

\section{INTRODUCTION}

Multi-antenna or Multiple-Input-Multiple-Output (MIMO) systems are attractive in point-to-point and ad-hoc networks as they are more robust to channel variations and can increase data rates through multiplexing and coherent signal combining. Multi-antenna systems can also suppress network interference by both receivers and transmitters (e.g. see [2]).

Potential MIMO system performance depends on the Channel-State-Information (CSI) available at the Tx and Rx side of links. For instance, independent equal-power streams should be transmitted on each antenna to achieve capacity in Additive-White-Gaussian-Noise channels with Rx CSI and no Tx CSI. With Tx CSI, capacity is achieved by parallelizing the channel between the Tx and Rx using a Singular-ValueDecomposition (SVD) of the channel matrix (see e.g. [3]). Most references assume Rx CSI since receivers can estimate channel parameters from signals received from transmitters.

Here, we assume that receivers know the covariance matrix of the interference at their antennas which could be accurately estimated using blind techniques such as the sampleinterference-covariance matrix. Transmitters know the channel parameters only between themselves and their target receivers which we refer to as Tx Link CSI. Tx Link CSI can be estimated with minimal overhead in slowly-varying, duplex systems with reciprocity since transmitters can use channel estimates acquired when they acted as receivers.

$\dagger$ Dan Bliss is currently working at MIT Lincoln Laboratory (bliss@1l.mit.edu). This research was supported in part by the National Science Foundation under Grant ANI-0333902. Portions of this material have appeared in [1]. Opinions, interpretations, conclusions, and recommendations are those of the authors and are not necessarily endorsed by the United States Government.
We present a technique to find the asymptotic spectral efficiency of an interference-limited representative link with an arbitrary distribution of interference powers, and interference due to spatially distributed nodes. We assume that each transmitter sends $M$ data streams with arbitrary correlation between the power allocated to each stream for any given node. Limiting the number of transmit data streams is known to increase network spectral efficiency as shown analytically in [4], [5] for systems without Tx CSI, and by simulation for systems with Tx CSI in [6].

Also, [4] found that transmitters should transmit a single data stream from one antenna in the high interference regime and independent data streams from each antenna in low interference. In [7], the authors found that the mean network spectral efficiency is bounded from above by a function which remains constant if the number of receiver antennas increases linearly with the number of transmitters. Neither [4] nor [7] model the spatial distribution of nodes, which is key to understanding large wireless networks since signal and interference strengths depend on relative node locations. Spatially distributed wireless networks with multiple-antenna links and no Tx CSI were studied in [5], which found the asymptotic spectral efficiency of a representative link as the number of receiver antennas $N$ and interferers in the network $n$ tend to infinity. The mean spectral efficiency was found to be a function of $N /\left(\rho r_{1}^{2}\right)$ where $\rho$ is the interferer density and $r_{1}$ the length of a representative link. [8] characterized the spectral efficiency of spatially distributed wireless networks with limited Tx CSI where transmitters use beam-forming to focus a single signal stream on their target receiver which uses spatial matched filtering (MF). In contrast, we assume multiple transmit streams per user and a Minimum-Mean-Square-Error (MMSE) receiver, which is optimal for Gaussian distributed transmit signals. We use an asymptotic analysis to address the added complexity of the MMSE receiver over the MF.

Our results characterize the spectral efficiency of multiantenna links in ad-hoc wireless networks as a function of tangible parameters such as link length, node density, number of antennas and path-loss exponent and facilitate trade-offs between spectral efficiency and the increased hardware costs of using more antennas or transmit data streams. Additionally, these results enable us to determine the spectral efficiency gains that Tx Link CSI provides. 


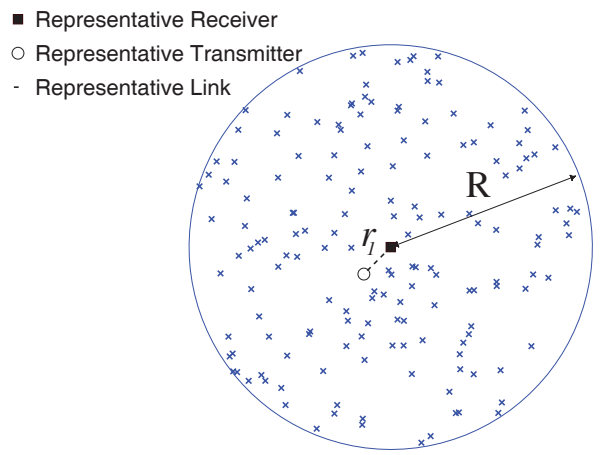

Fig. 1: Illustration of wireless network with representative link.

\section{System MOdEL}

\section{A. General Ad-hoc Network}

Consider a wireless network where there are $n+1$ receivers and $n+1$ transmitters where each transmitter is linked to a single receiver. Let $\mathcal{R}_{i}$ denote the $i$-th receiver and $\mathcal{T}_{i}$ denote the $i$-th transmitter. Consider a representative link (link-1) between $\mathcal{R}_{1}$ and $\mathcal{T}_{1}$ where $\mathcal{T}_{i}$ for $i=2,3, \cdots n+1$ are cochannel interferers to the representative link.

Each $\mathcal{T}_{i}$ has $K \leq N$ antennas and $\mathcal{R}_{1}$ has $N$. We assume frequency-flat fading where the channel between the $j$-th antenna of $\mathcal{T}_{i}$ and $k$-th antenna $\mathcal{R}_{1}$ is modeled as $\sqrt{\gamma_{i}} g_{i j k}$ where $\gamma_{i}$ is the path-loss between $\mathcal{T}_{i}$ and $\mathcal{R}_{1}$ and $g_{i j k}$ are IID, zero-mean, unit-variance complex Gaussian random variables whose PDF is denoted by $\mathcal{C N}(0,1)$. We make the standard assumption that nodes transmit using Gaussian code-books. Each transmitting node knows the channel coefficients between itself and its target receiver, but not to any other nodes. The receivers know the channel coefficients between themselves and their target transmitters and the spatial covariance matrix of the interference, i.e. the covariance of the interference at their $N$ antennas. Additionally, we assume a noise power of $N \bar{\sigma}^{2}$ at each antenna of the representative receiver. The factor $N$ is required to avoid singularities in the asymptotic analysis which applies in the regime as $n, N \rightarrow \infty$ with the ratio of interferers to receiver antennas $c=n M / N>0$. All limiting results refer to this regime.

\section{B. Spatially Distributed Network}

Consider a circular network of radius $R$ with $n$ wireless transmitters at random independent, identically distributed (IID) points in the circle such that $n=\rho \pi R^{2}$.

The representative receiver $\mathcal{R}_{1}$ is assumed to be at the origin of the circle and $\mathcal{T}_{1}$ is at a distance $r_{1}$ from $\mathcal{R}_{1}$ as shown in Figure 1. The $n$ interferers are in links with other receivers whose locations do not impact the representative link. Let $r_{i}$ denote the distance between transmitting node $i$ and the origin. The path-loss $\gamma_{i}=G_{t} r_{i}^{-\alpha}$ with $\alpha>2$ and $G_{t}$ a proportionality constant, which is a standard model for spatially distributed networks.

For technical reasons, we assume a noise power $N \bar{\sigma}^{2}$ where:

$$
\bar{\sigma}^{2}=\sigma^{2}\left(N^{-\frac{\alpha}{2}}\right)
$$

where $\sigma^{2}$ is a constant. This assumption enables the asymptotic analysis of the SINR as $N \rightarrow \infty$. Without this assumption, as $N \rightarrow \infty$, the system will no longer be interferencelimited as the MMSE receiver will reduce interference to levels comparable to the noise. Since we focus on the interferencelimited regime in this work, we assume that $\sigma^{2}$ is small and does not significantly effect the spectral efficiency.

\section{Parallelized System}

\section{A. Parallelized Transmissions with Link CSI}

Since transmitters don't have CSI of unintended receivers, they cannot transmit in directions that reduce interference. Hence, it is optimal for nodes to parallelize the channels between themselves and their targets using an SVD and transmit independent data streams on each parallelized channel with some power allocation [4]. We assume that all transmitters transmit on the $M$ parallelized channels with the strongest singular values. Let $P_{i j}$ for $i=1,2, \cdots n+1$ and $j=1,2, \cdots, M$ denote the power allocated to the $j$-th stream by the $i$-th transmitter. Let the $P_{i j}$ be IID for over $i$, i.e. the power allocations of a given transmitter are independent of other transmitters. For a given transmitter, there can be arbitrary correlation between the transmit powers it allocates to its $M$ streams. Let the PDF and CDF of $P_{i j}$ for all $i$ and each $j$ be denoted by $f_{j}(x)$ and $F_{j}(x)$, respectively, and the total transmit power per node be bounded by $P_{M}$.

Let the $N \times K$ matrix $\mathbf{H}_{i j}$ contain the channel coefficients between the antennas of $\mathcal{T}_{i}$ and $\mathcal{R}_{j}$. Let $\lambda_{i j}$ denote the $j$-th largest singular value of $\mathbf{H}_{i 1}$ and $\mathbf{v}_{i j}$ be the corresponding right singular vector of $\mathbf{H}_{i 1}$. The transmit signal of $\mathcal{T}_{1}$ is thus $\mathbf{x}=\left[\mathbf{v}_{11} \cdots \mathbf{v}_{1 M}\right] \tilde{\mathbf{x}}$ where $\tilde{\mathbf{x}}$ is an $M \times 1$ vector of IID data samples with $E\left[\tilde{\mathbf{x}} \tilde{\mathbf{x}}^{\dagger}\right]=\operatorname{diag}\left(P_{11}, \cdots, P_{1 M}\right)$ and $\mathbf{x}$ is a $K \times 1$ vector of transmit samples.

For large $N$, the spectral efficiency of link-1 can be approximated as:

$$
\begin{aligned}
& C_{1} \approx \\
& \sum_{j=1}^{M} \log _{2}\left(1+\gamma_{1} P_{1 j} \lambda_{1 j} \mathbf{u}_{1 j}^{\dagger}\left(N \bar{\sigma}^{2} \mathbf{I}+\mathbf{K}_{1} \boldsymbol{\Phi}_{1} \mathbf{K}_{1}^{\dagger}\right)^{-1} \mathbf{u}_{1 j}\right)
\end{aligned}
$$

with $\mathbf{K}_{1}$ an $N \times n M$ matrix of $\mathcal{C N}(0,1)$ entries,

$$
\begin{aligned}
\boldsymbol{\Phi}_{1}= & \operatorname{diag}\left(\gamma_{2} P_{21}, \cdots, \gamma_{2} P_{2 M}, \gamma_{3} P_{31},\right. \\
& \left.\cdots, \gamma_{3} P_{3 M}, \cdots \gamma_{n+1} P_{(n+1) 1}, \cdots, \gamma_{n+1} P_{(n+1) M}\right)
\end{aligned}
$$

and $\mathbf{u}_{1 j}$ are unit-norm isotropic vectors.

\section{B. Asymptotic Spectral Efficiency of the Parallelized System}

The spectral efficiency of the parallelized system described in the previous sub-section is characterized by the following theorem. Note that Theorem 1 is presented for the general assumptions in Section II-A and does not rely on the spatial distribution of nodes.

Theorem 1: Let $\Psi_{N}(\tau)$ denote the CDF of the pathlosses from the interferers to the representative receiver $\gamma_{2}, \gamma_{3}, \cdots, \gamma_{n+1}$. Assume that as $n, N \rightarrow \infty$ with the ratio 
of the number of interferers to receiver antennas $n M / N=$ $c$ and $\Psi_{N}(\tau) \rightarrow \Psi(\tau)$ either uniformly or such that $\int \Psi_{N}(x / \tau) d \tau<\infty$ for all $N$ and $x$. Then the spectral efficiency of link-1 in (2) converges with probability 1 to

$$
C_{1}^{*}=\sum_{j=1}^{M} \log _{2}\left(1+\lambda_{j}^{*} P_{1 j} \gamma_{1} \beta\right)
$$

where $\beta$ is a unique, non-negative solution to the equation:

$$
-\bar{\sigma}^{2} \beta+1=\beta c \int_{0}^{\infty} \frac{x d H(x)}{1+x \beta}
$$

where $H(x)$ is the limiting empirical distribution of interference powers from all streams of all interferers given by:

$$
H(x)=\frac{1}{M} \sum_{j=1}^{M} \int f_{j}(\tau) \Psi(x / \tau) d \tau
$$

and $\lambda_{j}^{*}$ is the limiting value of the $j$-th eigenvalue of a Wishart matrix $\frac{1}{N} \mathbf{G G}^{\dagger}$ where $\mathbf{G}$ is an $N \times K$ matrix with IID $\mathcal{C N}(0,1)$ entries. In particular if $N, K \rightarrow \infty$ such that $K / N=d>0$

$$
\lambda_{1}^{*}=\lambda_{2}^{*}=\cdots=\lambda_{M}^{*}=(1+\sqrt{d})^{2}
$$

and if $K$ is a finite constant,

$$
\lambda_{1}^{*}=\lambda_{2}^{*}=\cdots=\lambda_{M}^{*}=1 .
$$

Proof: The proof is based on convergence properties of functions of random variables (e.g. see [9]) and Lemma 1 on the convergence with probability 1 of the SINR.

Lemma 1: Consider the SINR of the $j$-th data stream from the representative transmitter at the output of the representative receiver given by the following expression (e.g. [10]):

$$
\operatorname{SINR}_{j}=\gamma_{1} P_{1 j} \lambda_{1 j} \mathbf{u}_{1 j}^{\dagger}\left(N \bar{\sigma}^{2} \mathbf{I}+\mathbf{K}_{1} \boldsymbol{\Phi}_{1} \mathbf{K}_{1}^{\dagger}\right)^{-1} \mathbf{u}_{1 j}
$$

Under the assumptions of Theorem $1, \mathrm{SINR}_{j}$ converges with probability 1 to an asymptotic limit given by:

$$
\gamma_{1} P_{1 j} \lambda_{j}^{*} \beta
$$

where $\beta$ is a unique, non-negative solution for $\beta$ in (5) and where $\lambda_{j}^{*}$ are defined in Theorem 1 .

Proof: This lemma can be proved using the main result of [11] and the isotropic property of $\mathbf{u}_{1 j}$.

Note that Lemma 1 is closely related to several results in the literature such as [11] and [12] which show the convergence of the SINR for random CDMA or systems with receiver antenna diversity. The main difference between these results and Lemma 1 is that the other works do not apply when the vector $\mathbf{u}_{1 j}$ in (9) is isotropic and of unit norm as they require the entries of $\mathbf{u}_{1 j}$ to have a greater degree of independence.

Additionally, we note that the convergence of $\lambda_{1}, \lambda_{2}, \cdots, \lambda_{M}$ to either 1 or $(1+\sqrt{d})^{2}$ is slow and is not a good approximation for moderate values of $N$ and $K$. Instead, we propose the following approximation which is based on the asymptotic distribution of the e.d.f. of the eigenvalues of Wishart matrices denoted by $F_{d}(x)$ which is known to be:

$$
\lambda_{1 j}^{*} \approx F_{d}^{-1}((N-j+1) / N) .
$$

For $K / N=d=1$,

$$
F(x)= \begin{cases}\frac{\pi+\sqrt{4 x-x^{2}}+2 \arcsin \left(-1+\frac{1}{2} x\right)}{2 \pi} & \text { if } 0 \leq x<4 \\ 1 & \text { otherwise }\end{cases}
$$

and $F^{-1}(x)$ can be evaluated numerically.

\section{Application to Spatially Distributed NETWORKS}

\section{A. Asymptotic Spectral Efficiency}

We apply the results of the previous section to the spatially distributed network model of Section II-B. In this case it is known from [5] that as $N \rightarrow \infty$, the SINR in the interferencelimited regime for systems without Tx CSI grows as $N^{\alpha / 2}$. To avoid singularities, we define a normalized SINR for the $j$-th data stream from the representative receiver as follows:

$$
\eta_{N}^{j}=N^{-\alpha / 2} \operatorname{SINR}_{j} .
$$

This normalization is accomplished by scaling the path-loss of the interferers and the thermal noise by $N^{\alpha / 2}$ and finding the limiting SINR for this new system using Lemma 1 which gives the limiting normalized SINR.

Theorem 2: As the number of interferers $n$, receiver antennas $N$ and network radius $R \rightarrow \infty$ with $n M / N=c$ and $n=\pi \rho R^{2}$, the normalized SINR for stream- $i, \eta_{N}^{i}$ approaches an asymptotic limit with probability 1 as follows:

$$
\eta_{N}^{i} \rightarrow \eta^{i}=P_{1 i} \lambda_{i}^{*} G_{t} r_{1}^{-\alpha} \beta
$$

where $\beta$ satisfies the following equation:

$$
\begin{gathered}
\frac{2 \pi^{2} \rho\left(G_{t} \beta\right)^{\frac{2}{\alpha}}}{\alpha} \sum_{j=1}^{M} E\left[P_{j}^{\frac{2}{\alpha}}\right] \csc \left(\frac{2 \pi}{\alpha}\right)-\frac{2 \pi \rho \beta}{\alpha} \times \\
\int_{0}^{\infty} \frac{\tau^{-\frac{2}{\alpha}}}{1+\tau \beta} d \tau \sum_{j=1}^{M} \int_{\tau / b}^{\infty} f_{j}(x) x^{\frac{2}{\alpha}} d x+\beta \sigma^{2}=1
\end{gathered}
$$

where $b=\left(\frac{\pi \rho N}{n}\right)^{\alpha / 2}$, and $E\left[P_{j}^{2 / \alpha}\right]$ is the expected value of the transmit power allocated by the interferers to their $j$-th strongest stream, raised to the power $\frac{2}{\alpha}$.

Proof: This theorem can be proved by combining Theorem 1 with the techniques used to prove Theorem 1 in [5].

The relationship of parameters such as the SINR, number of receiver antennas, interferer density and path-loss are not clear from (15). A few approximations can be made to yield additional insight into how the various factors contribute to the limiting SINR. From Lemma 7 of [1], we note that if $n / N$ is very large, i.e. the number of nodes in the network is much larger than the number of antennas per receiver, the transmit power limit per node implies that the second term on the LHS of (15) is small. Furthermore, we assume that the 
thermal noise power is small which implies that the third term on the LHS of (15) is small. Using these approximations:

$$
\begin{aligned}
& \frac{2 \pi^{2} \rho\left(G_{t} \beta\right)^{\frac{2}{\alpha}}}{\alpha} \sum_{j=1}^{M} E\left[P_{j}^{\frac{2}{\alpha}}\right] \csc \left(\frac{2 \pi}{\alpha}\right) \approx 1 \\
& \beta \approx \frac{1}{G_{t}}\left[\frac{\alpha}{2 \pi^{2} \rho \sum_{j=1}^{M} E\left[P_{j}^{\frac{2}{\alpha}}\right]} \sin \left(\frac{2 \pi}{\alpha}\right)\right]^{\alpha / 2}
\end{aligned}
$$

which when subtituted into (14) yields:

$$
\eta_{i} \approx \lambda_{1 i}^{*} P_{1 i}\left[\frac{\alpha}{2 \pi^{2} \rho r_{1}^{2} \sum_{j=1}^{M} E\left[P_{j}^{\frac{2}{\alpha}}\right]} \sin \left(\frac{2 \pi}{\alpha}\right)\right]^{\alpha / 2}
$$

Writing $G_{\alpha}=\left(\frac{\alpha}{2 \pi} \sin \left(\frac{2 \pi}{\alpha}\right)\right)^{\alpha / 2}$, rescaling the normalized SINR by $N^{\alpha / 2}$ and summing the contribution from $M$ streams yields the following approximation for the spectral efficiency of link-1 for large $N$ :

$$
C_{1} \approx \sum_{i=1}^{M} \log _{2}\left(1+\lambda_{1 i}^{*} P_{1 i} G_{\alpha}\left[\frac{N}{\pi \rho r_{1}^{2} \sum_{j=1}^{M} E\left[P_{j}^{\frac{2}{\alpha}}\right]}\right]^{\frac{\alpha}{2}}\right)
$$

We illustrate the utility of (18) using two models for transmit power. In the equal-power model, the transmit power used on all streams by the interferers are constants $P$. In the second model which we call the 2-class model, class 1 nodes transmit with power $P_{1}$ on all streams, and class- 2 nodes transmit with power $P_{2}$ from a single stream. The classes are assigned independently with class 1 occuring with probability $q$.

For the equal-power model, (18) becomes:

$$
C_{1}^{e p} \approx \sum_{i=1}^{M} \log _{2}\left(1+\lambda_{1 i}^{*} G_{\alpha}\left[\frac{N}{M \pi \rho r_{1}^{2}}\right]^{\frac{\alpha}{2}}\right) .
$$

For the 2-class model with Link-1 assigned to the first class:

$$
C_{1}^{2 c} \approx \sum_{i=1}^{M} \log _{2}\left(1+\lambda_{1 i}^{*} P_{1} G_{\alpha}\left[\frac{N}{\pi \rho r_{1}^{2} \bar{P}}\right]^{\alpha / 2}\right)
$$

where $\bar{P}=\left(q M P_{1}^{\frac{2}{\alpha}}+(1-q) P_{2}^{\frac{2}{\alpha}}\right)$.

\section{B. Monte-Carlo Simulations}

To validate (19) and (20), we simulated 1000 interferers at random locations in a circle with $10^{-3}$ nodes per unit area. The path-loss exponent $\alpha=4$, and the thermal noise level was set to a constant $\bar{\sigma}^{2}=1 \times 10^{-13} \mathrm{~W}$. Note that the specific value of $\bar{\sigma}^{2}$ does not play a significant role in the interference-limited systems we simulated. In each case the number of antennas at the representative receiver $N$ and the transmitting nodes $K$ were equal, and 1000 trials were conducted for each $N$.

For the equal power model, transmit power per-stream for each node $P=\frac{1}{M} \mathrm{~W}$ on each of the $M$ data streams. For the two-class model, each interferer was class 1 with probability $q=0.5$, where class 1 interferers transmitted with $P_{1}=0.5 \mathrm{~W}$

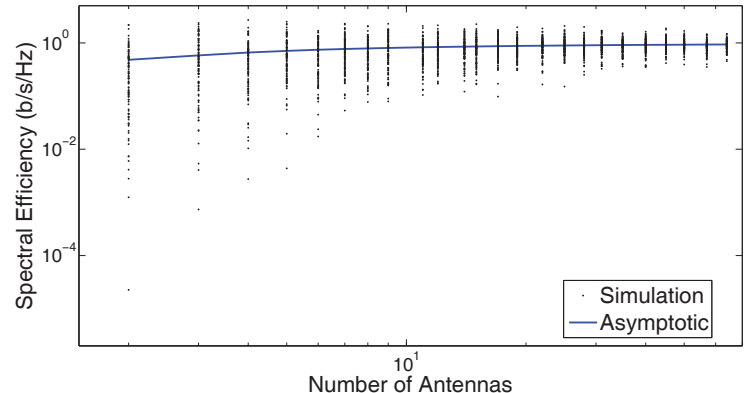

Fig. 2: Spectral efficiency vs. number of antennas for spatially distributed networks with constant transmit powers and two streams.

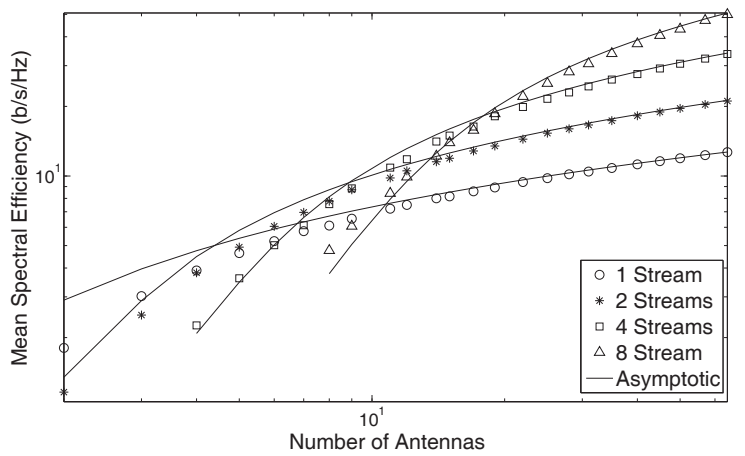

Fig. 3: Mean spectral efficiency vs. number of antennas for spatially distributed networks with constant transmit powers.

on each of $M$ streams and class 2 interferers transmitted $P_{2}=$ $1 \mathrm{~W}$ on a single stream. The representative transmitter was always class 1 . For both models, we simulated $M=1,2,4$ and 8 streams per transmitting node with equal numbers of antennas at all transmitters, and the representative receiver.

Figure 2 shows results of simulations of systems for which the spectral efficiency is normalized by scaling the interference powers by $N^{\alpha / 2}$ for the equal-power model and $M=2$. The solid lines indicate the normalized spectral efficiency, and the points represent simulation results. (Note that for clarity, we plotted a random sampling of 100 of the 1000 trials that were conducted). The normalized spectral efficiencies clearly converge to the asymptotic limit as indicated by the concentration of points with increasing $N$.

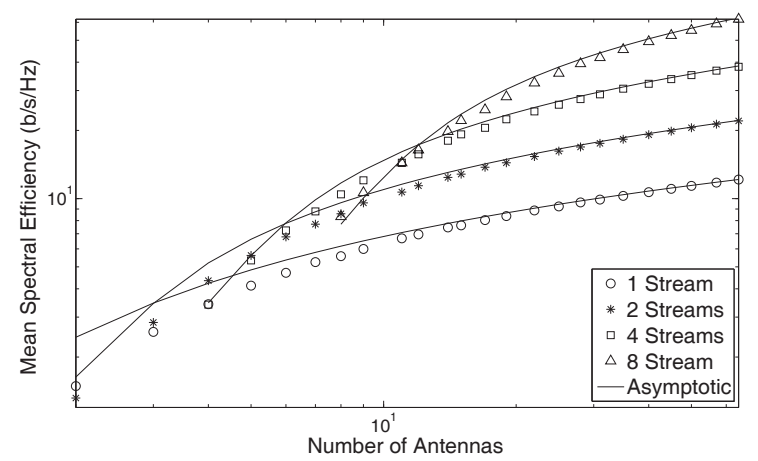

Fig. 4: Mean spectral efficiency vs. number of antennas for spatially distributed networks with the two-class model. 


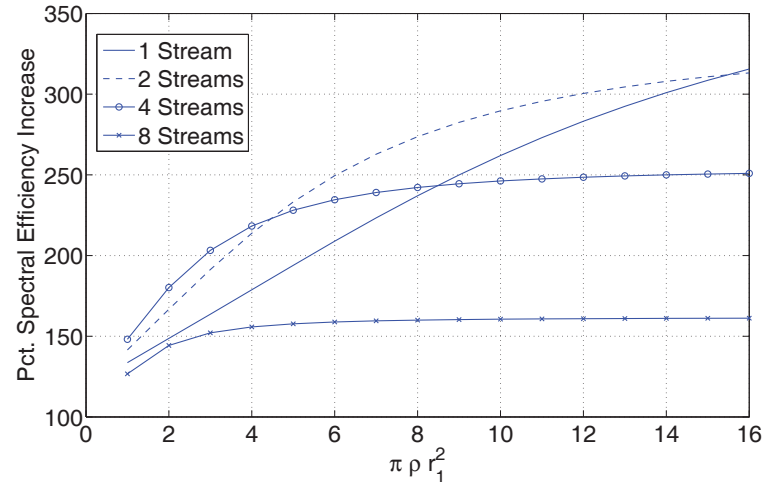

Fig. 5: Percentage increase in mean spectral efficiency with Link Tx CSI over systems with no Tx CSI vs. $\pi \rho r_{1}^{2}$ for $N=12$ antennas per node.

The un-normalized spectral efficiencies given by (19) and (20) do not converge as the spectral efficiency increases with $N$. To verify these equations, we plotted the average simulated spectral efficiencies for the equal-power and two-class models along with (19) and (20) respectively. Figures 3 and 4 show the simulated mean spectral efficiencies for the equal-power and 2class models respectively. For the equal transmit power model, Figure 3 indicates that the asymptotic expression is within $10 \%$ of the simulated mean spectral efficiencies when the number of antennas is 11 or greater in all cases simulated. For the twoclass model, the simulated mean spectral efficiency agrees to within $10 \%$ of the asymptotic expression when $N \geq 9$ as shown in Figure 4.

\section{Comparison with Systems without Transmit CSI}

We plotted the ratio of the asymptotic mean spectral efficiency of systems with and without Tx Link CSI to analyze its benefits. If the transmit power allocated to each stream is $P$, the mean spectral efficiency without Tx CSI is [5]:

$$
E\left[C_{1}^{n c}\right] \approx M \log _{2}\left(1+G_{\alpha}\left[\frac{N}{A M}\right]^{\frac{\alpha}{2}}\right)
$$

Figure 5 shows the percentage increase in mean spectral efficiency with Tx Link CSI vs. the quantity $A=\pi \rho r_{1}^{2}$ which can be interpreted as the average number of interferers closer to the receiver than its target transmitter and was defined as the link rank in [5]. Note that the spectral efficiency gain with Tx Link CSI is highly dependent on $A$. For instance, for $A=6$ and two transmit streams, Tx Link CSI provides a two-fold increase in spectral efficiency, with a greater than three-fold increase for higher link ranks. This indicates that a significant (but not orders of magnitude) increase in spectral efficiency is possible with link Tx CSI, which can be acquired with low overhead in duplex systems with reciprocity.

\section{Summary AND CONCLUSIONS}

A technique to find the asymptotic spectral efficiency of multi-antenna links in ad-hoc wireless networks where transmitters have CSI corresponding to their desired receivers is presented. Transmitters are restricted to using $M<\infty$ channel modes, and the results are asymptotic in the regime where the number of receiver antennas and interferers go to infinity with a constant ratio. The asymptotic predictions are supported by numerical simulations.

In spatially distributed networks, the asymptotic spectral efficiency which approximates the mean spectral efficiency for relatively small numbers of antennas, is found to be dependent on the ratio of interferer density to the number of receiver antennas as given in (18). Thus, as is the case for systems without Tx CSI [5], it is possible to maintain a constant mean spectral efficiency per link if the number of antennas per receiver is increased linearly with interferer density.

Additionally, we found that the spectral efficiency in the network can be increased in certain cases if each transmitter transmits fewer data streams. Compare this to MIMO links in AWGN channels where nodes should transmit data on all their channel modes with a water-filling power allocation. Figure 3 illustrates this fact as the asymptotic spectral efficiency with fewer streams is actually higher than the asymptotic spectral efficiency with larger number of streams when the number of antennas is small. Note that a similar observation was made in [5] for systems without Tx CSI.

We find that the asymptotic spectral efficiency with Tx Link CSI can be several times higher than without Tx CSI. The benefit of using Tx Link CSI increases for longer links, denser networks, or both, as illustrated by Figure 5. For instance, with 12 antennas at transmitters and receivers, and 4 transmit streams, Tx CSI can double the spectral efficiency when the quantity $\pi \rho r_{1}^{2}=3$. Since Tx Link CSI can be estimated in duplex systems with reciprocity without a significant increase in overhead, Tx Link CSI can be useful, particularly in dense networks with long links.

\section{REFERENCES}

[1] S. Govindasamy, Multiple-antenna Systems in Ad-Hoc Wireless Networks, PhD dissertation, Massachusetts Institute of Technology, 2008.

[2] D. W. Bliss and K. W. Forsythe, "Information theoretic comparison of MIMO wireless communication receivers in the presence of interference," Proc. 38th Asilomar Conference on Signals, Systems and Computers, 2004.

[3] D. Tse and P. Viswanath, Fundamentals of Wireless Communication, Cambridge University Press, 2005.

[4] R. S. Blum, "MIMO capacity with interference," IEEE J. Sel. Areas of Comm., vol. 21, no. 5, pp. 793-801, June 2003.

[5] S. Govindasamy, D. W. Bliss, and D. H. Staelin, "Spectral efficiency in single-hop ad-hoc wireless networks with interference using adaptive antenna arrays," IEEE J. on Sel. Areas of Comm., Sept. 2007.

[6] M. F. Demirkol and M.A. Ingram, "Stream control in networks with interfering MIMO links," IEEE WCNC, Mar. 2003.

[7] B. Chen and M. J. Gans, "MIMO communications in ad-hoc networks," IEEE Trans. Signal Processing, vol. 54, pp. 2773-2783, July 2006.

[8] A. M. Hunter, J. G. Andrews, and S. Weber, "Transmission capacity of ad hoc networks with spatial diversity," IEEE Trans. on Wireless Comm., 2009.

[9] Alan F. Karr, Probability, Springer-Verlag, 1993.

[10] H.L. Van Trees, Optimum Array Processing, John Wiley \& Sons, 2002.

[11] Z.D. Bai and J. W. Silverstein, "On the signal-to-interference-ratio of CDMA systems in wireless communications," Annals of Applied Probability, vol. 17, no. 1, pp. 81-101, 2007.

[12] S. Hanly and D. Tse, "Resource pooling and effective bandwidths in CDMA systems with multiuser receivers and spatial diversity," IEEE Trans. on Inform. Theory, vol. 47, no. 4, pp. 1328-1351, May 2001. 